\title{
MMSE Extension of V-BLAST based on Sorted QR Decomposition
}

\author{
Dirk Wübben, Ronald Böhnke, Volker Kühn, and Karl-Dirk Kammeyer \\ Department of Communications Engineering \\ University of Bremen \\ Otto-Hahn-Allee, D-28359 Bremen, Germany \\ Email: \{wuebben, boehnke, kuehn, kammeyer\}@ant.uni-bremen.de
}

\begin{abstract}
In rich-scattering environments layered space-time architectures like the BLAST system may exploit the capacity advantage of multiple antenna systems. In this paper, we present a novel, computationally efficient algorithm for detecting V-BLAST architectures with respect to the MMSE criterion. It utilizes a sorted QR decomposition of the channel matrix and leads to a simple successive detection structure. The new algorithm needs only a fraction of computational effort compared to the standard V-BLAST algorithm and achieves the same error performance.

Index Terms-BLAST, MIMO systems, Zero-Forcing and MMSE detection, wireless communication.
\end{abstract}

\section{INTRODUCTION}

In rich-scattering environments the V-BLAST (Vertical Bell Labs Layered Space-Time) architecture proposed in [1] exploits the capacity advantage of multiple antenna systems. It uses a vertically layered coding structure, where independent code blocks (called layers) are associated with a particular transmit antenna. At the receiver, these layers are detected by a successive interference cancellation technique which nulls the interferers by linearly weighting the received signal vector with a zero-forcing nulling vector (ZF-BLAST). This successive detection requires multiple calculations of pseudoinverses, being a computational expensive task. A reduced complexity detection algorithm utilizing a sorted QR decomposition of the channel matrix was proposed by the authors in [2], [3]. It jointly calculates an optimized detection order and the QR decomposition of the channel matrix and is called ZF-SQRD (ZF Sorted QR Decomposition). An adaption of the original ZF-BLAST to the MMSE criterion was presented in [4] and a version with lower complexity was introduced in [5].

In this paper, we extend the ZF-SQRD algorithm to the MMSE solution, called MMSE-SQRD. Similar to ZF-SQRD it does not always find the optimal detection order and from there a performance degradation may occur. If this drawback is not acceptable for the specific application, a post-sorting algorithm (PSA) can be used, leading to the ideal detection sequence and thus to the performance of MMSE-BLAST. However, the combination of MMSE-SQRD and PSA requires only a fraction of computational effort compared to the BLAST detection algorithm.

This work was supported in part by the German ministry of education and research $(\mathrm{BMBF})$ under grant $01 \mathrm{BU} 153$.
The remainder of this paper is as follows. In Section II, the system model and notation is introduced. In order to simplify later derivation we recall the linear ZF and MMSE filter and introduce an extended system model in Section III. The detection of BLAST systems using the QR decomposition of the channel matrix is investigated in Section IV. The computational effort and the performance analysis are given in Section V and VI, respectively. Concluding remarks can be found in Section VII.

\section{SYSTEM DESCRIPTION}

We consider a multiple antenna system with $n_{T}$ transmit and $n_{R} \geq n_{T}$ receive antennas. The data is demultiplexed into $n_{T}$ data substreams of equal length (called layers). These substreams are optionally encoded by a convolutional code (CC), bit-interleaved, mapped onto $M$-PSK or $M$-QAM symbols $s_{i}$ and transmitted over the $n_{T}$ antennas simultaneously. For simplicity we will assume uncoded substreams for the derivation of the detection algorithms, but will investigate the performance of coded and uncoded systems in Section VI.

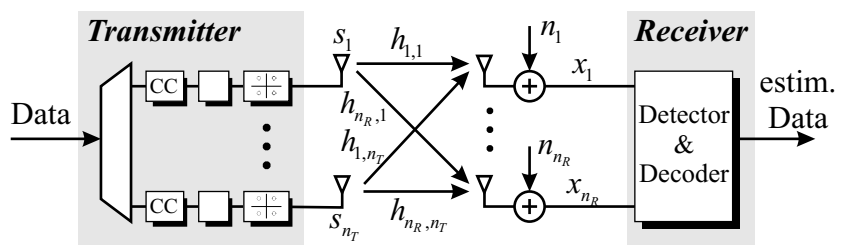

Fig. 1. Model of a MIMO system with $n_{T}$ transmit and $n_{R}$ receive antennas.

In order to describe the MIMO system, one time slot of the time-discrete complex baseband model is investigated. Let $^{1} \mathbf{s}=\left[s_{1} \ldots s_{n_{T}}\right]^{T}$ denote the $n_{T} \times 1$ transmit signal vector, then the corresponding $n_{R} \times 1$ receive signal vector $\mathbf{x}=\left[x_{1} \ldots x_{n_{R}}\right]^{T}$ is given by

$$
\mathbf{x}=\mathbf{H} \mathbf{s}+\mathbf{n} .
$$

In (1), $\mathbf{n}=\left[n_{1} \ldots n_{n_{R}}\right]^{T}$ represents white gaussian noise of variance $\sigma_{n}^{2}$ observed at the $n_{R}$ receive antennas while

${ }^{1}$ Throughout this paper, $(\cdot)^{T}$ and $(\cdot)^{H}$ denote matrix transpose and hermitian transpose, respectively. Furthermore, $\mathbf{I}_{\alpha}$ indicates the $\alpha \times \alpha$ identity matrix and $\mathbf{0}_{\alpha, \beta}$ denotes the $\alpha \times \beta$ all zero matrix. 
the average transmit power of each antenna is normalized to one, i.e. $\mathrm{E}\left\{\mathbf{s s}^{H}\right\}=\mathbf{I}_{n_{T}}$ and $\mathrm{E}\left\{\mathbf{n n}^{H}\right\}=\sigma_{n}^{2} \mathbf{I}_{n_{R}}$. The $n_{R} \times n_{T}$ channel matrix $\mathbf{H}$ contains uncorrelated complex gaussian fading gains with unit variance. We assume a flat fading environment, where the channel matrix $\mathbf{H}$ is constant over a frame and changes independently from frame to frame (block fading channel). The distinct fading gains are assumed to be perfectly known by the receiver.

In order to detect the transmitted information, it would be optimal to use a maximum-likelihood (ML) detector. As the computational effort is of order $M^{n_{T}}$, ML detection is not feasible for larger number of transmit antennas or higher modulation schemes. Therefore, we present suboptimal detection schemes with reduced complexity in the following sections.

\section{LINEAR DETECTION}

In this section we recall the linear detection with respect to the zero-forcing $(\mathrm{ZF})$ and to the minimum-mean-squareerror (MMSE) criterion. By introducing an extended system model, we show the similarity of both criteria. This analogy will play a key role for the introduction of the MMSE based QR detection algorithm in Section IV.

\section{A. Zero-Forcing Detector $(Z F)$}

In a linear detector, the receive signal vector $\mathbf{x}$ is multiplied with a filter matrix $\mathbf{G}$, followed by a parallel decision on all layers. Zero-forcing means that the mutual interference between the layers shall be perfectly suppressed. This is accomplished by the Moore-Penrose pseudo-inverse (denoted by $(\cdot)^{+}$) of the channel matrix [6]

$$
\mathbf{G}_{\mathrm{ZF}}=\mathbf{H}^{+}=\left(\mathbf{H}^{H} \mathbf{H}\right)^{-1} \mathbf{H}^{H},
$$

where we assumed that $\mathbf{H}$ has full column rank. The decision step consists of mapping each element of the filter output vector

$$
\tilde{\mathbf{s}}_{\mathrm{ZF}}=\mathbf{G}_{\mathrm{ZF}} \mathbf{x}=\mathbf{H}^{+} \mathbf{x}=\mathbf{s}+\left(\mathbf{H}^{H} \mathbf{H}\right)^{-1} \mathbf{H}^{H} \mathbf{n}
$$

onto an element of the symbol alphabet by a minimum distance quantization. The estimation errors of the different layers correspond to the main diagonal elements of the error covariance matrix

$$
\mathbf{\Phi}_{\mathrm{ZF}}=\mathrm{E}\left\{\left(\tilde{\mathbf{s}}_{\mathrm{ZF}}-\mathbf{s}\right)\left(\tilde{\mathbf{s}}_{\mathrm{ZF}}-\mathbf{s}\right)^{H}\right\}=\sigma_{n}^{2}\left(\mathbf{H}^{H} \mathbf{H}\right)^{-1}
$$

which equals the covariance matrix of the noise after the receive filter. It is obvious that small eigenvalues of $\mathbf{H}^{H} \mathbf{H}$ will lead to large errors due to noise amplification. This effect is especially observed in systems with equal number of transmit and receive antennas. In fact, using a result from random matrix theory [7], it can be shown that in the large system limit for $n_{T}=n_{R} \rightarrow \infty$ the noise amplification tends to infinity almost surely. In order to improve the performance the noise term can be included in the design of the filter matrix G. This is done by the MMSE detection scheme, where the filter represents a trade-off between noise amplification and interference suppression.

\section{B. MMSE Detector}

The MMSE detector minimizes the mean squared error (MSE) between the actually transmitted symbols and the output of the linear detector and leads to the filter matrix [6]

$$
\mathbf{G}_{\text {MMSE }}=\left(\mathbf{H}^{H} \mathbf{H}+\sigma_{n}^{2} \mathbf{I}_{n_{T}}\right)^{-1} \mathbf{H}^{H} .
$$

The resulting filter output is given by

$$
\tilde{\mathbf{s}}_{\mathrm{MMSE}}=\mathbf{G}_{\mathrm{MMSE}} \mathbf{x}=\left(\mathbf{H}^{H} \mathbf{H}+\sigma_{n}^{2} \mathbf{I}_{n_{T}}\right)^{-1} \mathbf{H}^{H} \mathbf{x} .
$$

The estimation errors of the different layers correspond to the main diagonal elements of the error covariance matrix

$$
\begin{aligned}
\boldsymbol{\Phi}_{\text {MMSE }} & =\mathrm{E}\left\{\left(\tilde{\mathbf{s}}_{\text {MMSE }}-\mathbf{s}\right)\left(\tilde{\mathbf{s}}_{\text {MMSE }}-\mathbf{s}\right)^{H}\right\} \\
& =\sigma_{n}^{2}\left(\mathbf{H}^{H} \mathbf{H}+\sigma_{n}^{2} \mathbf{I}_{n_{T}}\right)^{-1} .
\end{aligned}
$$

With the definition of a $\left(n_{T}+n_{R}\right) \times n_{T}$ extended channel matrix $\underline{\mathbf{H}}$ and $\mathrm{a}\left(n_{T}+n_{R}\right) \times 1$ extended receive vector $\underline{\mathbf{x}}$ through

$$
\underline{\mathbf{H}}=\left[\begin{array}{c}
\mathbf{H} \\
\sigma_{n} \mathbf{I}_{n_{T}}
\end{array}\right] \quad \text { and } \quad \underline{\mathbf{x}}=\left[\begin{array}{c}
\mathbf{x} \\
\mathbf{0}_{n_{T}, 1}
\end{array}\right] \text {, }
$$

the output of the MMSE filter given by (6) can be rewritten as

$$
\tilde{\mathbf{s}}_{\text {MMSE }}=\left(\underline{\mathbf{H}}^{H} \underline{\mathbf{H}}\right)^{-1} \underline{\mathbf{H}}^{H} \underline{\mathbf{x}}=\underline{\mathbf{H}}^{+} \underline{\mathbf{x}} \text {. }
$$

Furthermore, the error covariance matrix (7) becomes

$$
\boldsymbol{\Phi}_{\mathrm{MMSE}}=\sigma_{n}^{2}\left(\underline{\mathbf{H}}^{H} \underline{\mathbf{H}}\right)^{-1}=\sigma_{n}^{2} \underline{\mathbf{H}}^{+} \underline{\mathbf{H}}^{+H} \text {. }
$$

Comparing (9) and (10) to the corresponding expression for linear zero-forcing detector in (3) and (4), the only difference is that the channel matrix $\mathbf{H}$ has been replaced by $\underline{\mathbf{H}}$. This observation is extremely important for incorporating the MMSE criterion into the SQRD based detection algorithm.

\section{BLAST DETECTION}

The V-BLAST detection algorithm [1] bases on the linear zero-forcing solution, but detects the signals one after another and not in parallel. In order to achieve the best performance, it is optimal to choose always the layer with the largest post detection signal-to-noise-ratio (SNR), or equivalently with the smallest estimation error. The adaptation to the MMSE criterion was presented in [4], [5], where the optimal sequence maximizes the signal-to-interference-and-noise ratio (SINR) in each detection step. The main drawback of the V-BLAST detection algorithms lies in the computational complexity, as it requires multiple calculations of the pseudo-inverse of the channel matrix [3].

\section{A. Zero-Forcing BLAST with QR Decomposition}

It was shown in several publications, e.g. [2], [3], [8], that the ZF-BLAST algorithm can be restated in terms of the QR decomposition of the channel matrix $\mathbf{H}=\mathbf{Q R}$, where the $n_{R} \times n_{T}$ matrix $\mathbf{Q}$ has orthogonal columns with unit norm and the $n_{T} \times n_{T}$ matrix $\mathbf{R}$ is upper triangular. Multiplying the received signal $\mathbf{x}$ with $\mathbf{Q}^{H}$ yields the sufficient statistic

$$
\tilde{\mathbf{s}}=\mathbf{Q}^{H} \mathbf{x}=\mathbf{R} \mathbf{s}+\boldsymbol{\eta}
$$


for the estimation of transmit vector $\mathbf{s}$. As $\mathbf{Q}$ is an unitary matrix, the statistical properties of the noise term $\boldsymbol{\eta}=\mathbf{Q}^{H} \mathbf{n}$ remain unchanged. Due to the upper triangular structure of $\mathbf{R}$, the $k$-th element of $\tilde{\mathbf{s}}$ is given by

$$
\tilde{s}_{k}=r_{k, k} \cdot s_{k}+\sum_{i=k+1}^{n_{T}} r_{k, i} \cdot s_{i}+\eta_{k}
$$

and is free of interference from layers $1, \ldots, k-1$. Thus, $\tilde{s}_{n_{T}}$ is totally free of interference and can be used to estimate $s_{n_{T}}$ after appropriate scaling with $1 / r_{n_{T}, n_{T}}$. Proceeding with $\tilde{s}_{n_{T}-1}, \ldots, \tilde{s}_{1}$ and assuming correct previous decisions, the interference can be perfectly cancelled in each step. Then it follows from (12) that the SNR of layer $k$ is determined by the diagonal element $\left|r_{k, k}\right|^{2}$.

As already mentioned, the detection sequence is crucial due to the risk of error propagation. It can be modified by permuting elements of $\mathbf{s}$ and the corresponding columns of $\mathbf{H}$ prior to the QR decomposition, leading to different matrices $\mathbf{Q}$ and $\mathbf{R}$ [3]. In order to find the optimum sequence, $\left|r_{k, k}\right|$, which represents the component of the column vector $\mathbf{h}_{k}$ that is perpendicular to the space spanned by $\mathbf{h}_{1}, \ldots, \mathbf{h}_{k-1}$, needs to be maximized for $k=n_{T}, \ldots, 1$. This may be accomplished in a straight forward way by performing $\mathcal{O}\left(n_{T}^{2} / 2\right)$ QR decompositions of permutations of $\mathbf{H}$ [9]. The heuristic approach ZF-SQRD does not assure the optimal order and therefore leads to a small performance degradation but with only of a fraction of computational complexity [2], [3]. After introducing the QR based MMSE detection, we will present the extension of ZF-SQRD to the MMSE criterion.

\section{B. MMSE QR Detection}

In order to extend the QR based detection with respect to the MMSE criterion, we can apply the similarity of ZF and MMSE detection noted in Section III-B. We introduce the QR decomposition of the extended channel matrix (8)

$$
\underline{\mathbf{H}}=\left[\begin{array}{c}
\mathbf{H} \\
\sigma_{n} \mathbf{I}_{n_{T}}
\end{array}\right]=\underline{\mathbf{Q}} \underline{\mathbf{R}}=\left[\begin{array}{c}
\mathbf{Q}_{1} \\
\mathbf{Q}_{2}
\end{array}\right] \underline{\mathbf{R}}=\left[\begin{array}{c}
\mathbf{Q}_{1} \underline{\mathbf{R}} \\
\mathbf{Q}_{2} \underline{\mathbf{R}}
\end{array}\right],
$$

where the $\left(n_{T}+n_{R}\right) \times n_{T}$ matrix $\underline{\mathbf{Q}}$ with orthonormal columns was partitioned into the $n_{R} \times n_{T}$ matrix $\mathbf{Q}_{1}$ and the $n_{T} \times n_{T}$ matrix $\mathbf{Q}_{2}$. Obviously,

$$
\underline{\mathbf{Q}}^{H} \underline{\mathbf{H}}=\mathbf{Q}_{1}^{H} \mathbf{H}+\sigma_{n} \mathbf{Q}_{2}^{H}=\underline{\mathbf{R}}
$$

holds and from the relation $\sigma_{n} \mathbf{I}_{n_{T}}=\mathbf{Q}_{2} \underline{\mathbf{R}}$ it follows that

$$
\underline{\mathbf{R}}^{-1}=\frac{1}{\sigma_{n}} \mathbf{Q}_{2},
$$

i.e. the inverse $\underline{\mathbf{R}}^{-1}$ is a byproduct of the $\mathrm{QR}$ decomposition and $\mathbf{Q}_{2}$ is an upper triangular matrix. This relation will be useful for the post-sorting algorithm proposed in Section IVD. Using (15) and (14), the filtered receive vector becomes

$$
\tilde{\mathbf{s}}=\underline{\mathbf{Q}}^{H} \underline{\mathbf{x}}=\mathbf{Q}_{1}^{H} \mathbf{x}=\underline{\mathbf{R}} \mathbf{s}-\sigma_{n} \mathbf{Q}_{2}^{H} \mathbf{s}+\mathbf{Q}_{1}^{H} \mathbf{n} .
$$

The second term on the right hand side of (16) including the lower triangular matrix $\mathbf{Q}_{2}^{H}$ constitutes the remaining interference that can not be removed by the successive interference cancellation procedure. This points out the trade-off between noise amplification and interference suppression.

The optimum detection sequence now maximizes the signalto-interference-and-noise ratio (SINR) for each layer, leading to minimal estimation error for the corresponding detection step. The estimation errors of the different layers in the first detection step correspond to the diagonal elements of the error covariance matrix (10)

$$
\boldsymbol{\Phi}=\sigma_{n}^{2}\left(\underline{\mathbf{H}}^{H} \underline{\mathbf{H}}\right)^{-1}=\sigma_{n}^{2} \underline{\mathbf{R}}^{-1} \underline{\mathbf{R}}^{-H} .
$$

The estimation error after perfect interference cancellation is given by $\sigma_{n}^{2} /\left|\underline{r}_{k, k}\right|^{2}$. Thus, it is again optimal to choose the permutation that maximizes $\left|\underline{r}_{k, k}\right|$ in each detection step. The algorithm proposed in the next section determines an optimized detection sequence within a single sorted $\mathrm{QR}$ decomposition and thereby significantly reduces the computational complexity in comparison to standard MMSE-BLAST algorithms.

\section{MMSE Sorted QR Decomposition (MMSE-SQRD)}

In order to obtain the optimal detection order, first $\left|\underline{r}_{n_{T}, n_{T}}\right|$ has to be maximized over all possible permutations of the columns of the extended channel matrix $\underline{\mathbf{H}}$, followed by $\left|\underline{r}_{n_{T}-1, n_{T}-1}\right|$, and so on. Unfortunately, using standard algorithms for the QR decomposition, the diagonal elements of $\underline{\mathbf{R}}$ are calculated just in the opposite order, starting with $\underline{r}_{1,1}$. This makes finding the optimal order of detection a difficult task.

A heuristic approach of arranging the order of detection into the QR decomposition for the ZF detection was presented in [2], [3]. This sorted QR decomposition algorithm is basically an extension to the modified Gram-Schmidt procedure by reordering the columns of the channel matrix prior to each orthogonalization step. In the sequel we present an adapted version of this algorithm for MMSE detection.

The fundamental idea is that $\left|\underline{r}_{k, k}\right|$ is minimized in the order it is computed $\left(1, \ldots, n_{T}\right)$ instead of being maximized in the order of detection $\left(n_{T}, \ldots, 1\right)$. This is motivated by the fact that the layers detected last affect only few other layers through error propagation and may therefore have rather small SINR's, which increases the probability of large SINR's in the first layers. Now, $\underline{r}_{1,1}$ is simply the norm of the column vector $\underline{\mathbf{h}}_{1}$, so the first optimization in the SQRD algorithm consists merely of permuting the column of $\underline{H}$ with minimum norm to this position. During the following orthogonalization of the vectors $\underline{\mathbf{h}}_{2}, \ldots, \underline{\mathbf{h}}_{n_{T}}$ with respect to the normalized vector $\underline{\mathbf{h}}_{1}$, the first row of $\underline{\mathbf{R}}$ is obtained. Next, $\underline{\boldsymbol{r}}_{2,2}$ is determined in a similar fashion from the remaining $n_{T}-1$ orthogonalized vectors, et cetera. Thereby, the extended channel matrix $\underline{\mathbf{H}}$ is successively transformed into the matrix $\underline{\mathbf{Q}}$ associated with the desired ordering, while the corresponding $\underline{\mathbf{R}}$ is calculated row by row. Note that the column norms have to be calculated only once in the beginning and can be easily updated afterwards. Hence, the computational overhead due to sorting is negligible. An in-place-description of the whole MMSE-SQRD algorithm 
is given in Tab. $\mathbf{1}$, with $\underline{\mathbf{q}}_{i}$ indicating column $i$ of $\mathbf{Q}$ and vector $\mathbf{p}$ denoting the permutation of the columns of $\mathbf{H}$.

Tab. 1 MMSE-SQRD ALGORITHM

(1) $\underline{\mathbf{R}}=\mathbf{0}, \mathbf{Q}=\underline{\mathbf{H}}, \mathbf{p}=\left(1, \ldots, n_{T}\right)$

(2) for $i=1, \ldots, n_{T}$

(3) $\quad \operatorname{norm}_{i}=\left\|\underline{\mathbf{q}}_{i}\right\|^{2}$

(4) end

(5) for $i=1, \ldots, n_{T}$

(6) $k_{i}=\arg \min _{\ell=i, \ldots, n_{T}}$ norm

(7) exchange columns $i$ and $k_{i}$ in $\underline{\mathbf{R}}, \mathbf{p}$, norm and in the first $n_{R}+i-1$ rows of $\mathbf{Q}$

(8) $r_{i, i}=\sqrt{\text { norm }_{i}}$

(9) $\quad \underline{\mathbf{q}}_{i}:=\underline{\mathbf{q}}_{i} / \underline{r}_{i, i}$

(10) for $k=i+1, \ldots, n_{T}$

(11) $\quad r_{i, k}=\underline{\mathbf{q}}_{i}^{H} \cdot \underline{\mathbf{q}}_{k}$

(12) $\quad \underline{\mathbf{q}}_{k}:=\underline{\mathbf{q}}_{k}-\underline{r}_{i, k} \cdot \underline{\mathbf{q}}$

(13) $\quad \operatorname{norm}_{k}:=\operatorname{norm}_{k}-\underline{r}_{i, k}^{2}$

(14) end

(15) end

It should be emphasized that MMSE-SQRD does not always lead to the perfect detection sequence, but in many cases of interest the performance degradation is small compared to the reduced complexity. Furthermore, whenever MMSE-SQRD fails to find the optimal order, the post-sorting algorithm described in the sequel may be applied. It assures the optimal sorting and thereby achieves the same performance as MMSEBLAST.

\section{Post-Sorting-Algorithm (PSA)}

In order to introduce the Post-Sorting-Algorithm (PSA), we investigate the structure of the error covariance matrix in case of optimal sorting in more detail. Due to the relation (15) the error covariance matrix (17) is given by

$$
\mathbf{\Phi}=\mathbf{Q}_{2} \mathbf{Q}_{2}^{H}
$$

and $\mathbf{Q}_{2}$ is a square root of $\boldsymbol{\Phi}$ [5]. As $\mathbf{Q}_{2}$ is upper triangular, the $k$-th diagonal element of $\boldsymbol{\Phi}$ is proportional to the norm of the $k$-th row of $\mathbf{Q}_{2}$. Recalling the optimal ordering criterion, the last row of $\mathbf{Q}_{2}$ must have minimum norm of all rows. Assume that this condition is fulfilled, then the last row of the upper left $n_{T}-1 \times n_{T}-1$ submatrix of $\mathbf{Q}_{2}$ must have minimum norm of all rows of this submatrix. In case of the correct sorting this condition is accomplished by all upper left submatrices.

Now assume that this condition is not fulfilled for the matrix $\mathbf{Q}_{2}$. Then the row with minimum norm and the last row (as well as the corresponding elements of $\mathbf{p}$ ) need to be exchanged at the expense of destroying the upper triangular structure. However, by right multiplying the permuted version of $\mathbf{Q}_{2}$ with a proper unitary $n_{T} \times n_{T}$ Householder reflection matrix ${ }^{2}$

\footnotetext{
${ }^{2}$ The Householder matrix for a $1 \times n$ row vector a with complex elements is given by $\mathbf{\Theta}=\mathbf{I}_{n}-(1+w) \mathbf{u}^{H} \mathbf{u}$ with the definitions $\mathbf{u}=\frac{\mathbf{a}-\|\mathbf{a}\| \mathbf{e}_{n}}{\|\mathbf{a}-\| \mathbf{a}\left\|\mathbf{e}_{n}\right\|}$, $\mathbf{e}_{n}=\left[\mathbf{0}_{1, n-1} 1\right]$ and $w=\frac{\mathbf{u a}^{H}}{\mathbf{a u}^{H}}$. Thus, $\mathbf{a} \Theta=\left[\mathbf{0}_{1, n-1}\|\mathbf{a}\|\right]$ holds.
}

$\boldsymbol{\Theta}$, a block triangular matrix is achieved. Finally, $\mathbf{Q}_{1}$ has to be updated to $\mathbf{Q}_{1} \boldsymbol{\Theta}$. Instead of permuting columns of $\underline{\mathbf{R}}$ and left multiplying with $\Theta^{H}$ in each step, we can alternatively invert $\mathbf{Q}_{2}$ at the end of the PSA, due to the relation $\underline{\mathbf{R}}=1 / \sigma_{n} \mathbf{Q}_{2}^{-1}$.

These ordering and reflection steps are then iterated for the upper left $\left(n_{T}-1\right) \times\left(n_{T}-1\right)$ submatrix of the such modified matrix $\mathbf{Q}_{2}$ and the first $n_{T}-1$ columns of the new matrix $\mathbf{Q}_{1}$, resulting in the $\mathrm{QR}$ decomposition of the optimally ordered channel matrix $\underline{\mathbf{H}}$. The whole post-sorting algorithm is given in ${ }^{3}$ Tab. 2.

Tab. 2 POST-SORTING Algorithm

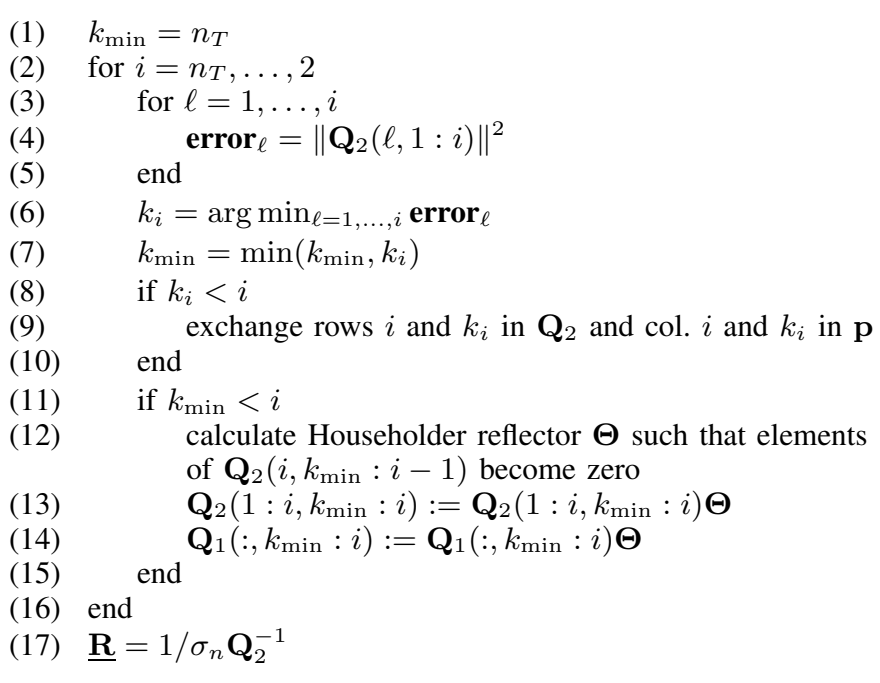

\section{Computational EfFort}

In this section we investigate the computational effort of the proposed sorting algorithm. Therefore, the complex floating point operations (flops) $f$ are specified according to the number of transmit and receive antennas. For simplicity, we count each addition as one flop and each multiplication as three flops. The MMSE-SQRD requires

$$
f_{\mathrm{SQRD}}=\frac{4}{3} n_{T}^{3}+4 n_{T}^{2} n_{R}+\frac{1}{3} n_{T}^{2}+2 n_{T} n_{R}+\frac{25}{6} n_{T}
$$

flops. The overhead in comparison to an unsorted QR decomposition is very small, only $2 n_{T}^{2}-2 n_{T}$ additional operations are necessary when the sorting steps are included in the decomposition. For the PSA the computational effort depends on the required number of permutations. We get an upper bound for the complexity by ignoring the upper triangular structure of $\mathbf{Q}_{2}$. In this case,

$f_{\mathrm{PSA}}=\frac{14}{3} n_{T}^{3}+4 n_{T}^{2} n_{R}+\frac{27}{2} n_{T}^{2}+3 n_{T} n_{R}+\frac{89}{6} n_{T}-7 n_{R}-30$ complex floating point operations are necessary. The computational effort of the Hassibi approach [5] can be approximated by $f_{\mathrm{SQRD}}+f_{\mathrm{PSA}}$. In case of MMSE-SQRD an optimized sorting is already given by the decomposition. Consequently, the PSA

\footnotetext{
${ }^{3} \mathbf{A}(a: b, c: d)$ denotes the submatrix of $\mathbf{A}$ with elements from rows $a, \ldots, b$ and columns $c, \ldots, d$.
} 
is only required in a fraction of all transmissions and therefore the complexity of the Hassibi approach serves as an upper bound for the expected overall complexity.

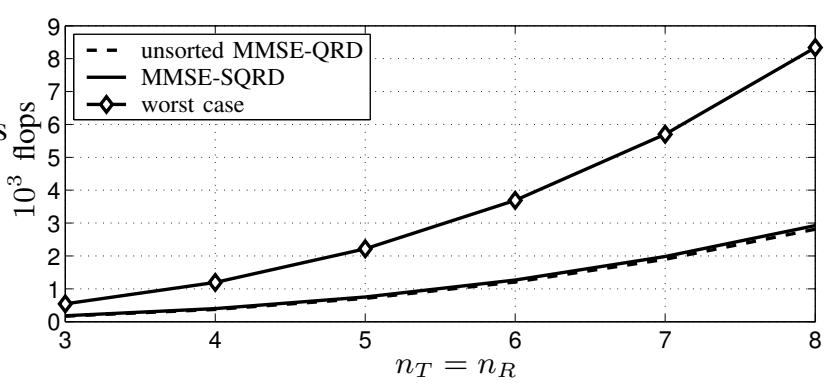

Fig. 2. Number of operations $f$ in flops for unsorted MMSE-QRD, MMSESQRD and the algorithm by Hassibi [5].

Fig. 2 shows the required number of complex floating point operations for the unsorted MMSE-QR decomposition, the SQRD and the Hassibi approach for varying number of $n_{T}=n_{R}$ antennas. Obviously the computational overhead of SQRD is extremely small and a significant reduction in comparison to the worst-case can be observed.

\section{Vi. Performance Analysis}

In this section, we investigate the frame error rates (FER) for a MIMO system with $n_{T}=4$ transmit and $n_{R}=4$ receive antennas and QPSK modulation. We compare uncoded data streams and encoded streams, where the half rate $(7,5)_{8}$ convolutional code was used in each layer. $E_{b}$ denotes the average energy per information bit arriving at the receiver, thus $E_{b} / N_{0}=n_{R} /\left(R_{c} \log _{2}(M) \sigma_{n}^{2}\right)$ holds. Fig. 3 shows the performance of MMSE-BLAST and MMSE-SQRD, both uncoded and coded.

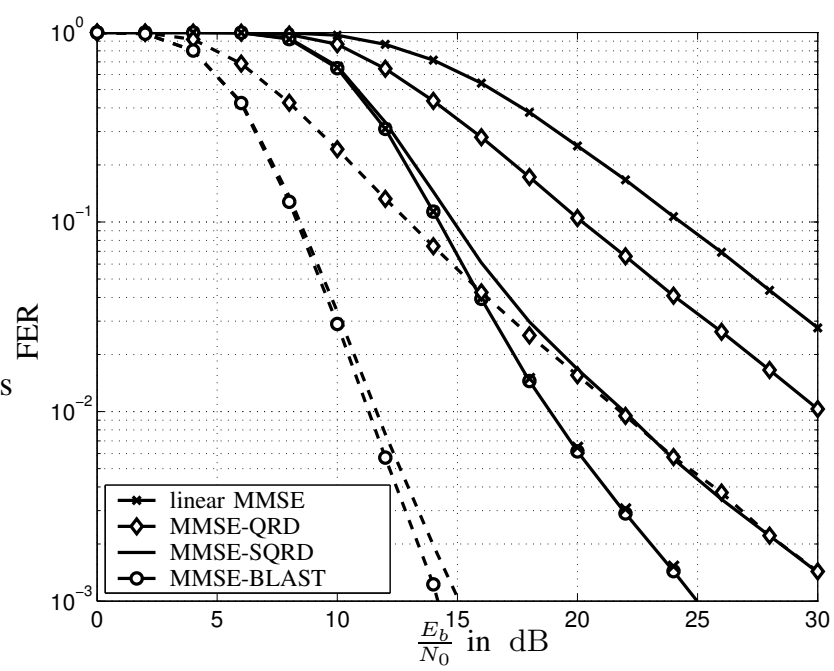

Fig. 3. Frame Error Rate of a system with $n_{T}=4$ and $n_{R}=4$ antennas, frame length $L=100$, QPSK symbols, uncoded (continuous line) and convolutional encoded (dotted line) substreams.

Comparing the simulation results of uncoded transmission, the successive detection schemes achieve an improved performance in comparison to the linear MMSE detector. The strong impact of ordering becomes obvious by comparing the unsorted (MMSE-QRD) and the schemes with optimized detection order. As the MMSE-SQRD does not assure the optimal order, a performance gap between MMSE-BLAST and MMSE-SQRD is observed. This gap is completely closed by applying the post-sorting algorithm, as MMSE-BLAST and MMSE-SQRD-PSA find the same detection sequence.

However, for the coded system the performance loss of MMSE-SQRD reduces to approximately $1 \mathrm{~dB}$ for a FER of $10^{-3}$ and is negligible for a FER of $10^{-2}$. On the other side, the gain in comparison to the coded MMSE-QRD is enormous. These observations can be explained in the following way. The sorting maximizes the minimum SINR of all layers [8] and thereby the operation point of the convolutional code is achieved. As the effect of error propagation is reduced by the application of forward error correction codes, the influence of suboptimal sorting decreases. Thus, in many cases of interest, the MMSE-SQRD would be the first choice for implementation due to the reduced complexity.

\section{SUMMARY AND CONCLUSIONS}

We have proposed a new detection algorithm for V-BLAST systems with respect to the MMSE criterion. The algorithm utilizes an optimized QR decomposition of the channel matrix and leads to a simple successive detection. For those cases, where MMSE-SQRD does not find the correct ordering, a reordering can easily be applied, thereby resulting in an optimum algorithm with reduced complexity. However, for coded transmission, the performance degradation of MMSESQRD compared to MMSE-BLAST is negligible.

\section{REFERENCES}

[1] P. W. Wolniansky, G. J. Foschini, G. D. Golden, and R. A. Valenzuela, "VBLAST: An Architecture for Realizing Very High Data Rates Over the Rich-Scattering Wireless Channel," in Proc. ISSE, Pisa, Italy, September 1998.

[2] D. Wübben, R. Böhnke, J. Rinas, V. Kühn, and K. D. Kammeyer, "Efficient Algorithm for Decoding Layered Space-Time Codes," IEE Electronics Letters, vol. 37, no. 22, pp. 1348-1350, October 2001.

[3] D. Wübben, J. Rinas, R. Böhnke, V. Kühn, and K. D. Kammeyer, "Efficient Algorithm for Detecting Layered Space-Time Codes," in Proc. ITG Conference on Source and Channel Coding, Berlin, Germany, January 2002, pp. 399-405.

[4] A. Benjebbour, H. Murata, and S. Yoshida, "Comparison of Ordered Successive Receivers for Space-Time Transmission," in Proc. IEEE Vehicular Technology Conference (VTC), USA, Fall 2001.

[5] B. Hassibi, "An Efficient Square-Root Algorithm for BLAST," in Proc. IEEE Intl. Conf. Acoustic, Speech, Signal Processing, Istanbul, Turkey, June 2000, pp. 5-9.

[6] S. Verdu, Muliuser Detection, 2nd ed. Cambridge, U.K.: Cambridge University Press, 1998.

[7] J. Silverstein and Z. Bai, "On the Empirical Distribution of Eigenvalues of a Class of Large Dimensional Random Matrices," Journal of Multivariate Analysis, vol. 54, no. 2, pp. 175-192, 1995.

[8] R. Böhnke, D. Wübben, V. Kühn, and K. D. Kammeyer, "Reduced Complexity MMSE Detection for BLAST Architectures," in Proc. IEEE Global Communications Conference (Globecom'03), San Francisco, California, USA, December 2003.

[9] G. J. Foschini, G. D. Golden, A. Valenzela, and P. W. Wolniansky, "Simplified Processing for High Spectral Efficiency Wireless Communications Emplying Multi-Element Arrays," IEEE Journal on Selected Areas in Commununications, vol. 17, no. 11, pp. 1841-1852, November 1999. 